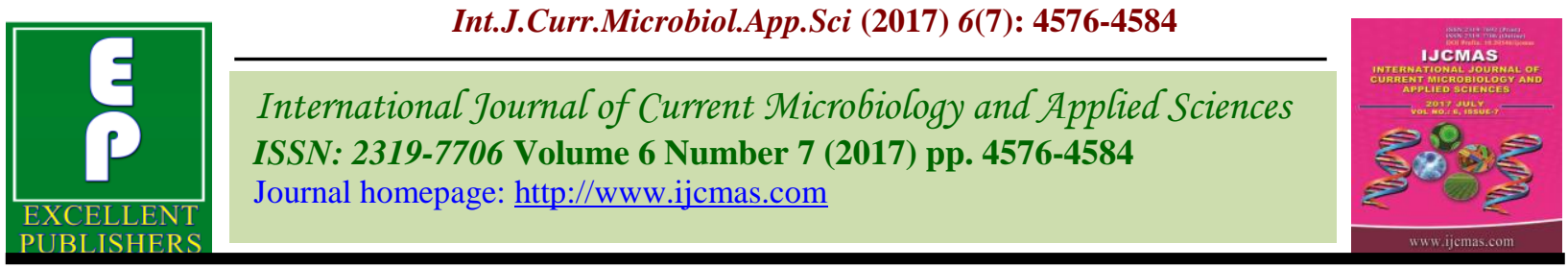

Original Research Article

https://doi.org/10.20546/ijcmas.2017.607.478

\title{
Influence of Set Row Cultivation on Pearlmillet-Sunflower Sequence Cropping System in Vertic-Inceptisol
}

\author{
G. S. Yadahalli ${ }^{*}$, M. B. Guled ${ }^{2}$ and V. S. Surakod ${ }^{3}$ \\ ${ }^{1}$ Department of Agronomy, UAS, Raichur, Karnataka, India \\ ${ }^{2}$ UHS, Bagalkot, Karnataka, India \\ ${ }^{3}$ AICRPDA, RARS, Vijayapura, UAS, Dharwad, Karnataka, India \\ *Corresponding author
}

A B S T R A C T

Keywords

Set row

cultivation,

Pearlmillet,

sunflower

cropping

sequence,

tank silt and microorganisms

Article Info

Accepted:

25 June 2017

Available Online:

10 July 2017

\begin{abstract}
A field experiment was carried out at to study the impact of set row cultivation on pearlmillet - sunflower crop sequence productivity and microbial population at AICRP on Dry land Agriculture, Regional Agricultural Research Station, Vijayapura, Karnataka during three consecutive kharif and rabi season. The pooled data indicated that, application of tank silt + crop residue in set furrows with paired row spacing (45-135-45 $\mathrm{cm})$ recorded significantly higher pearlmillet grain yield $(1737 \mathrm{~kg} / \mathrm{ha})$ over rest of the treatments. However, it was on par with the treatment receiving tank silt + crop residue in set furrows either with wider row $(135 \mathrm{~cm})$ or paired row planting techniques $(45-90-45$ $\mathrm{cm})$. In succeeding sunflower crop recorded higher sunflower yield $(717 \mathrm{~kg} / \mathrm{ha})$ with application of tank silt + crop residue in set furrows with wider row spacing $(135 \mathrm{~cm})$ followed by tank silt + crop residue in set furrows with paired row planting of 45-90-45 $\mathrm{cm}$ and 45-135-45 cm (674 and $694 \mathrm{~kg} / \mathrm{ha}$, respectively) and were significantly higher over farmers practice $(312 \mathrm{~kg} / \mathrm{ha})$. Similarly, considerable improvement in the population of soil micro-organisms was noticed. Application of tank silt and respective crop residues in set furrows with different planting patterns showed significantly higher population in bacteria, fungi, Actionmycetes, Azotobacter, PSB and Fluorescent Pseudomonas.
\end{abstract}

\section{Introduction}

Improvement in dry land agriculture holds the key to sustainable food and livelihood security for majority of the population in the country. The new paradigm for dryland agriculture calls for concurrent attention to the principles of ecology, economics, equity and employment.

Unscientific land and crop management practices have resulted in loss of nearly 6000 million tons of top fertile soil (16 t/ha/year) through erosion every year causing soil degradation in India. In Northern dry zone of Karnataka, occurrence of high runoff (20 to
$40 \%$ of annual rainfall), soil loss (12 to 43 t/ha/year) and nutrient loss (48 kg N, $16 \mathrm{~kg}$ $\mathrm{P}_{2} \mathrm{O}_{5}$ and $27 \mathrm{~kg} \mathrm{~K} 2 \mathrm{O} / \mathrm{ha}$ ) is a common feature (Guled et. al., 2003). Under dry land conditions adoption of appropriate soil and water conservation technique help to reduce runoff by 65 to 70 per cent and soil loss by 67 to 70 per cent. By adopting appropriate terrace level practices, soil loss and runoff can be minimized because these practices modify the land slope to desired grade when bunds are put at recommended vertical interval. On the other hand, adoption of suitable interterrace land management practices are 
known to minimize the soil loss, runoff and nutrient loss. Consequently, both the practices help in sustaining the higher yield level of crops, besides conserving the valuable soil and rainwater resources.

Set row cultivation is one of the in-situ moisture conservation practices. This system is widely followed by the farmers of Sourashtra region of Gujarat, where cultivation of crops in the wider rows, which are set permanently over long period in the dryland areas for higher crop productivity, is in practice.

It conserves rainwater effectively, improve the fertility of soil in set furrows and this technique offers an excellent drought proofing mechanism. Infiltration rate is increased effectively by reducing the runoff and soil loss. This practice will be able to provide the soil moisture at critical growth stages of the crops. Set row cultivation also improves soil physico-chemical and biological properties (Mishra, 2002).

\section{Materials and Methods}

A field experiment was conducted during three consecutive kharif and rabi season of $2004-05,2005$ - 06 and $2006-07$ on a fixed site to study the effect of set furrows on pearlmillet - sunflower crop productivity and microbial population at AICRP on Dryland Agriculture, Regional Agricultural Research Station, Vijayapura, Karnataka. Vertic Inceptisol are well drained medium black and the depth varied from $30-45 \mathrm{~cm}$ with excess lime nodules in the sub soils.

The soil of the experimental site was clay in texture with bulk density of $1.35 \mathrm{~g} / \mathrm{cc}, \mathrm{pH}$ of 8.11 with organic carbon content of $0.32 \%$. The soils were low in available $\mathrm{N}(154 \mathrm{~kg} / \mathrm{ha})$ and available $\mathrm{P}_{2} \mathrm{O}_{5}(16 \mathrm{~kg} / \mathrm{ha})$ and medium in available $\mathrm{K}_{2} \mathrm{O}(308 \mathrm{~kg} / \mathrm{ha})$. The experiment was laid out in a randomized complete block design with three replications. Application of tank silt@1886, 1650 and 1179 t/ha, respectively in $\mathrm{T}_{3}, \mathrm{~T}_{4}$ and $\mathrm{T}_{5}$ treatments in the initial period and respective organics were applied to the set furrows as per the treatment before sowing of the first crop in every year along with the recommended dose of fertilizer (50:25:0 kg and 35:50:35 kg N: $\mathrm{P}_{2} \mathrm{O}_{5}: \mathrm{K}_{2} \mathrm{O} / \mathrm{ha}$ for pearlmillet and sunflower, respectively) and FYM (7 t/ha) expect in farmer's practice (63 kg urea and $63 \mathrm{~kg} \mathrm{DAP} / \mathrm{ha})$.

The enumeration of total bacteria, fungi, actinomycetes, azotobacter, phosphate solubilizing bacteria and Fluorescent pseudomonas in the fresh soil samples was carried out by following the standard serial dilution plate count technique using Nutrient agar, Martin's Rose Bengal agar, Kuster's agar, Norrin - Nitrogen free glucose medium Pikovaskaya's medium and Kings B agar medium respectively.

\section{Results and Discussion}

\section{Pearlmillet yield}

Vertic-Inceptisols are characterized by low to very low available water holding capacity because of their lower depth. Consequently they support the crop growth for not more than 90 days.

Application of tank silt to a desired depth on the soil surface on entire land known to increase the available water holding capacity of such soils but it is very costly process. Hence, its application in set furrows is cheaper and affordable technique.

The technique of its application in set furrows was widely used in vertic-inceptisol of Northern Dry zone of Karnataka. In the present investigation, application of tank silt + crop residue in set furrows with paired row 
spacing (45-135-45 $\mathrm{cm})$ recorded significantly higher pearlmillet grain yield $(1737 \mathrm{~kg} / \mathrm{ha})$ over rest of the treatments. However, it was on par with the treatment receiving tank silt + crop residue in set furrows either with wider row $(135 \mathrm{~cm})$ or paired row planting techniques $(45-90-45 \mathrm{~cm})$ (Table 1).

The extent of yield increase with tank silt + crop residue application in set furrows was 77.24, 22.58, 21.38 and 20.37 per cent over farmers practice $(980 \mathrm{~kg} / \mathrm{ha})$, flatbed with wider row planting $(1417 \mathrm{~kg} / \mathrm{ha})$ and paired row spacing of $45-90-45 \mathrm{~cm}(1431 \mathrm{~kg} / \mathrm{ha})$ and 45-135-45cm (1443 kg/ha), respectively. Addition of tank silt or sediment to cultivated fields increases the crop growth and yield (Ramesh, 2001 and Binita, 2006). It is evident from the data that addition of tank silt + crop residue in set furrows with either paired row or wider row planting had conspicuous beneficial effect in achieving higher productivity. This increase in pearlmillet yield could be attributed to the presence of tank silt and crop residues in set furrows, which reduce the runoff and soil loss.

These crop residues in set furrows act as barrier and conserve the rain water, increase the infiltration rate, moisture retention capacity and nutrient availability in the soil. Improvement in soil moisture conservation by adopting of set row cultivation with soil amendments had also been reported by Mishra, (2002) and Anonymous, (2005).

\section{Microbial population}

The beneficial effect of crop residues on the soil organic carbon content and nutrient availability in turn influenced the nutrient uptake and crop yields. Degradation of crop residues in set furrows by soil microflora is often accelerated in the presence of soil fauna (Seastedt, 1984 and Tain et al., 1995). Soil microflora need regular maintenance of soil organic matter at higher level. In the present study, considerable improvement in the population of soil microflora was noticed. The bacteria, fungi, actinomycetes, azotobacter, PSB and Fluorescent pseudomonas population (pooled) were significantly higher with the application of tank silt + crop residue in set furrow with paired row spacing of 4590-45 cm (26.13 CFU/g of soil X10 ${ }^{7}, 16.16$, $14.78,19.77,29.65,25.14 \mathrm{CFU} / \mathrm{g}$ of soil $\mathrm{X} 10^{4}$, respectively) as compared to other treatments.

However, the superior treatment was on par with application of tank silt + crop residue in set furrows with wider and paired row spacing of $135 \mathrm{~cm}$ and $45-135-45 \mathrm{~cm}$. The increase in population was $70.34,130.2,113.8,139.0$ and 143.4 per cent, respectively over farmers' practice $(15.34,7.02,6.14,8.27,12.18$ and $10.84 \mathrm{cfu} / \mathrm{g}$ of soil) (Table 2 and 3 ). The soil organic carbon $(0.39 \%)$ level with addition of tank silt + crop residue and respective crop residue incorporation in set furrows played vital role for enhanced microbial activity.

Anonymous (1998) opined that residue incorporation in set row cultivation were found to be support more number of bacteria, fungi, actinomycetes, azotobacter and $\mathrm{P}$ solubilizers population. Incorporation of crop residues stimulated microorganism's population (Manna and Ganguly, 2001).

\section{Performance of succeeding sunflower yield}

Use of tank sediment, which is said to be available in plenty, is presumed to be a novel practice. However, making use of tank sediments, as an additive for the soil is said to supplement essential plant nutrients including micronutrients and improve soil physical, chemical and biological properties for sustained crop production (Keshavamurthy and Kotur, 1996). 
Table.1 Yield of pearlmillet and sunflower as influenced by the tank silt + crop residue application and Row spacing under set row cultivation (Pooled over 3 years)

\begin{tabular}{|c|c|c|c|c|c|c|c|c|}
\hline \multirow{2}{*}{ Treatment } & \multicolumn{4}{|c|}{ Pearlmillet yield (kg/ha) } & \multicolumn{4}{|c|}{ Sunflower yield (kg/ha) } \\
\hline & 2004 & 2005 & 2006 & Pooled & 2004-05 & $2005-06$ & 2006-07 & Pooled \\
\hline Farmers' practice $(35 \mathrm{~cm})$ & 997 & 947 & 997 & 980 & 260 & 158 & 517 & 312 \\
\hline Recommended practice $(60 \mathrm{~cm})$ & 1153 & 1180 & 1253 & 1196 & 387 & 255 & 650 & 430 \\
\hline Tank silt + CR in set furrows $(135 \mathrm{~cm})$ & 1493 & 1753 & 1853 & 1700 & 657 & 480 & 1013 & 717 \\
\hline Tank silt + CR in set furrows $(45-90-45 \mathrm{~cm})$ & 1500 & 1773 & 1873 & 1716 & 603 & 447 & 973 & 674 \\
\hline Tank silt + CR in set furrows $(45-135-45 \mathrm{~cm})$ & 1560 & 1745 & 1907 & 1737 & 620 & 464 & 997 & 694 \\
\hline Flatbed $(135 \mathrm{~cm})$ & 1227 & 1459 & 1567 & 1417 & 502 & 345 & 817 & 555 \\
\hline Flatbed $(45-90-45 \mathrm{~cm})$ & 1350 & 1457 & 1487 & 1431 & 463 & 297 & 793 & 518 \\
\hline Flatbed $(45-135-45 \mathrm{~cm})$ & 1280 & 1447 & 1603 & 1443 & 482 & 310 & 800 & 531 \\
\hline $\mathrm{S} . \mathrm{Em} \pm$ & 93 & 70 & 74 & 43 & 29 & 26 & 30 & 21 \\
\hline C.D at $5 \%$ & 281 & 213 & 225 & 129 & 88 & 79 & 90 & 62 \\
\hline
\end{tabular}

CR - Crop residue

Table.2 Soil microbial population at grand growth period of pearlmillet as influenced by the tank silt + crop residue application and Row spacing under set row cultivation (Pooled over 3 years)

\begin{tabular}{|c|c|c|c|c|c|c|c|c|c|c|c|c|}
\hline \multirow{2}{*}{ Treatment } & \multicolumn{4}{|c|}{ Bacteria (CFU /g soil X 10 ${ }^{7}$ ) } & \multicolumn{4}{|c|}{ Fungi (CFU /g soil X 10 ${ }^{4}$ ) } & \multicolumn{4}{|c|}{ Actinomycetes (CFU /g soil X 10 ${ }^{4}$ ) } \\
\hline & 2004 & 2005 & 2006 & Pooled & 2004 & 2005 & 2006 & Pooled & 2004 & 2005 & 2006 & Pooled \\
\hline Farmers' practice $(35 \mathrm{~cm})$ & 14.74 & 15.11 & 16.17 & 15.34 & 6.33 & 7.05 & 7.67 & 7.02 & 5.25 & 6.10 & 7.08 & 6.14 \\
\hline Recommended practice $(60 \mathrm{~cm})$ & 15.29 & 17.11 & 18.78 & $\mathbf{1 7 . 0 6}$ & 7.56 & 8.00 & 8.58 & 8.04 & 6.67 & 7.67 & 8.48 & 7.61 \\
\hline Tank silt + CR in set furrows $(135 \mathrm{~cm})$ & 20.22 & 24.30 & 29.53 & 24.68 & 11.87 & 14.66 & 17.42 & 14.65 & 10.73 & 13.86 & 14.81 & 13.13 \\
\hline Tank silt + CR in set furrows $(45-90-45 \mathrm{~cm})$ & 22.06 & 26.03 & 30.31 & 26.13 & 12.67 & 16.43 & 19.37 & 16.16 & 12.67 & 15.14 & 16.54 & 14.78 \\
\hline Tank silt + CR in set furrows $(45-135-45 \mathrm{~cm})$ & 20.78 & 25.11 & 29.69 & 25.19 & 13.11 & 15.11 & 18.56 & 15.59 & 11.66 & 14.33 & 14.96 & 13.65 \\
\hline Flatbed $(135 \mathrm{~cm})$ & 15.67 & 18.91 & 20.53 & 18.37 & 9.84 & 10.51 & 11.89 & $\mathbf{1 0 . 7 5}$ & 7.81 & 9.38 & 10.68 & 9.29 \\
\hline Flatbed $(45-90-45 \mathrm{~cm})$ & 17.28 & 20.40 & 21.65 & 19.78 & 10.25 & 11.67 & 11.20 & 11.07 & 8.67 & 10.67 & 11.41 & 10.25 \\
\hline Flatbed $(45-135-45 \mathrm{~cm})$ & 16.34 & 19.67 & 20.43 & 18.81 & 10.00 & 10.12 & 10.67 & 10.26 & 9.33 & 11.11 & 11.37 & 10.60 \\
\hline S. Em \pm & 0.91 & 0.84 & 1.24 & 0.72 & 0.50 & 0.80 & 0.90 & 0.44 & 0.63 & 0.65 & 0.80 & 0.47 \\
\hline C.D at $\overline{5 \%}$ & 2.76 & 2.55 & 3.77 & 2.18 & 1.53 & 2.42 & 2.73 & 1.35 & 1.92 & 1.98 & 2.43 & 1.42 \\
\hline
\end{tabular}

CR - Crop residue 
Table.2a Soil microbial population at grand growth period of pearlmillet as influenced by the tank silt + crop residue application and Row spacing under set row cultivation (Pooled over 3 years)

\begin{tabular}{|c|c|c|c|c|c|c|}
\hline Treatment & $\begin{array}{c}\text { Bacteria } \\
\text { (CFU/g of } \\
\text { soil X 10 })\end{array}$ & $\begin{array}{c}\text { Fungi } \\
(\mathrm{CFU} / \mathrm{g} \text { of } \\
\text { soil X 10 })\end{array}$ & $\begin{array}{c}\text { Actinomyce } \\
\text { tes (CFU/g } \\
\text { soil X 104) }\end{array}$ & $\begin{array}{c}\text { Azotobacte } \\
\text { r }(\text { CFU/g } \\
\text { soil X 10 } \text { 10 }^{4}\end{array}$ & $\begin{array}{c}\text { PSB } \\
\text { (CFU/g soil } \\
\text { X 10 })\end{array}$ & $\begin{array}{c}\text { FPs (CFU/g } \\
\text { soil X 104) }\end{array}$ \\
\hline Farmers' practice $(35 \mathrm{~cm})$ & 15.34 & 7.02 & 6.14 & 8.27 & 12.18 & 10.84 \\
\hline Recommended practice $(60 \mathrm{~cm})$ & 17.06 & 8.04 & 7.61 & 10.08 & 16.14 & 13.56 \\
\hline Tank silt + CR in set furrows $(135 \mathrm{~cm})$ & 24.68 & 14.65 & 13.13 & 18.07 & 27.41 & 23.72 \\
\hline Tank silt + CR in set furrows $(45-90-45 \mathrm{~cm})$ & 26.13 & 16.16 & 14.78 & 19.77 & 29.65 & 25.14 \\
\hline Tank silt + CR in set furrows $(45-135-45 \mathrm{~cm})$ & 25.19 & 15.59 & 13.65 & 18.87 & 28.55 & 24.39 \\
\hline Flatbed $(135 \mathrm{~cm})$ & 18.37 & 10.75 & 9.29 & 12.97 & 20.73 & 16.20 \\
\hline Flatbed $(45-90-45 \mathrm{~cm})$ & 19.78 & 11.07 & 10.25 & 14.46 & 22.32 & 17.93 \\
\hline Flatbed $(45-135-45 \mathrm{~cm})$ & 18.81 & 10.26 & 10.60 & 14.30 & 21.65 & 16.92 \\
\hline $\mathrm{S} . \mathrm{Em} \pm$ & 0.72 & 0.44 & 0.47 & 0.50 & 0.92 & 0.80 \\
\hline C.D at $5 \%$ & 2.18 & 1.35 & 1.42 & 1.51 & 2.79 & 2.44 \\
\hline
\end{tabular}

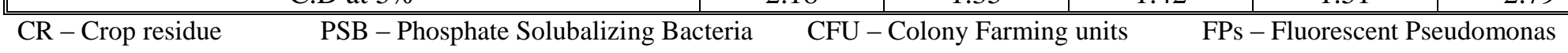

Table.3 Soil microbial population at grand growth period of pearlmillet as influenced by the tank silt + crop residue application and Row spacing under set row cultivation (Pooled over 3 years)

\begin{tabular}{|c|c|c|c|c|c|c|c|c|c|c|c|c|}
\hline \multirow{2}{*}{ Treatment } & \multicolumn{4}{|c|}{ Azotobacter (CFU /g soil X 10 $\left.{ }^{4}\right)$} & \multicolumn{4}{|c|}{ PSB (CFU /g soil X 10 $\left.{ }^{4}\right)$} & \multicolumn{4}{|c|}{ FPs $\left(\right.$ CFU /g soil X 10 $\left.{ }^{4}\right)$} \\
\hline & 2004 & 2005 & 2006 & Pooled & 2004 & 2005 & 2006 & Pooled & 2004 & 2005 & 2006 & Pooled \\
\hline Farmers' practice $(35 \mathrm{~cm})$ & 7.48 & 8.50 & 8.82 & 8.27 & 10.44 & 12.33 & 13.78 & 12.18 & 9.66 & 10.33 & 12.51 & 10.84 \\
\hline Recommended practice $(60 \mathrm{~cm})$ & 9.65 & 9.83 & 10.75 & 10.08 & 13.73 & 15.66 & 19.03 & 16.14 & 12.32 & 13.30 & 15.06 & 13.56 \\
\hline Tank silt + CR in set furrows $(135 \mathrm{~cm})$ & 16.63 & 17.89 & 19.69 & 18.07 & 23.86 & 28.33 & 30.05 & 27.41 & 20.79 & 23.89 & 26.48 & 23.72 \\
\hline Tank silt + CR in set furrows $(45-90-45 \mathrm{~cm})$ & 18.00 & 19.67 & 21.63 & 19.77 & 25.67 & 30.60 & 32.67 & 29.65 & 22.04 & 25.00 & 28.39 & 25.14 \\
\hline Tank silt + CR in set furrows $(45-135-45 \mathrm{~cm})$ & 17.66 & 18.33 & 20.61 & 18.87 & 24.70 & 29.11 & 31.84 & 28.55 & 21.61 & 24.21 & 27.34 & 24.39 \\
\hline Flatbed $(135 \mathrm{~cm})$ & 11.86 & 11.34 & 15.70 & 12.97 & 16.74 & 20.76 & 24.68 & 20.73 & 13.68 & 15.70 & 19.22 & 16.20 \\
\hline Flatbed $(45-90-45 \mathrm{~cm})$ & 12.67 & 13.67 & 17.03 & 14.46 & 18.00 & 22.17 & 26.80 & 22.32 & 15.37 & 17.33 & 21.08 & $\mathbf{1 7 . 9 3}$ \\
\hline Flatbed $(45-135-45 \mathrm{~cm})$ & 13.11 & 13.08 & 16.71 & 14.30 & 17.88 & 21.10 & 25.97 & 21.65 & 14.63 & 16.44 & 19.67 & 16.92 \\
\hline S. Em \pm & 1.04 & 0.65 & 0.85 & 0.50 & 0.93 & 0.99 & 1.04 & 0.92 & 0.91 & 0.70 & 1.21 & 0.80 \\
\hline C.D at $\overline{5 \%}$ & 3.15 & 1.98 & 2.59 & 1.51 & 2.81 & 3.00 & 3.15 & 2.79 & 2.76 & 2.13 & 3.68 & 2.44 \\
\hline
\end{tabular}

$\mathrm{CR}$ - Crop residue

PSB - Phosphate Solubalizing Bacteria

CFU - Colony Farming units

FPs - Fluorescent Pseudomonas 
Table.3a Soil microbial population at grand growth period of rabi sunflower as influenced by tank silt + crop residue application and Row spacing under set row cultivation (Pooled over 3 years)

\begin{tabular}{|c|c|c|c|c|c|c|}
\hline Treatment & $\begin{array}{c}\text { Bacteria } \\
\text { (CFU/g of soil } \\
\text { X 10 }) \\
\end{array}$ & $\begin{array}{c}\text { Fungi } \\
(\text { CFU/g of } \\
\left.\text { soil X 10 }{ }^{4}\right) \\
\end{array}$ & $\begin{array}{c}\text { Actionmyce } \\
\text { tes }(\mathrm{CFU} / \mathrm{g} \\
\text { soil X 10 }) \\
\end{array}$ & $\begin{array}{c}\text { Azotobacte } \\
\text { r (CFU/g } \\
\left.\text { soil X 10 }{ }^{4}\right) \\
\end{array}$ & $\begin{array}{c}\text { PSB } \\
\text { (CFU/g soil } \\
\text { X 10 } \text { 10 }^{4} \\
\end{array}$ & $\begin{array}{c}\text { FPs }(\text { CFU/g } \\
\left.\text { soil } X 10^{4}\right)\end{array}$ \\
\hline Farmers' practice $(35 \mathrm{~cm})$ & 12.13 & 8.27 & 6.65 & 8.27 & 12.18 & 10.84 \\
\hline Recommended practice $(60 \mathrm{~cm})$ & 13.73 & 10.26 & 8.30 & 10.08 & 16.14 & 13.56 \\
\hline Tank silt + CR in set furrows $(135 \mathrm{~cm})$ & 23.69 & 17.23 & 12.70 & 18.07 & 27.41 & 23.72 \\
\hline Tank silt + CR in set furrows $(45-90-45 \mathrm{~cm})$ & 24.77 & 18.35 & 13.74 & 19.77 & 29.65 & 25.14 \\
\hline Tank silt + CR in set furrows $(45-135-45 \mathrm{~cm})$ & 24.41 & 18.19 & 13.28 & 18.87 & 28.55 & 24.39 \\
\hline Flatbed $(135 \mathrm{~cm})$ & 16.59 & 11.94 & 9.32 & 12.97 & 20.73 & 16.20 \\
\hline Flatbed $(45-90-45 \mathrm{~cm})$ & 17.87 & 12.91 & 10.05 & 14.46 & 22.32 & 17.93 \\
\hline Flatbed $(45-135-45 \mathrm{~cm})$ & 17.29 & 12.30 & 9.68 & 14.30 & 21.65 & 16.92 \\
\hline $\mathrm{S} . \mathrm{Em} \pm$ & 0.64 & 0.43 & 0.30 & 0.57 & 0.92 & 0.80 \\
\hline C.D at $5 \%$ & 1.84 & 1.22 & 0.92 & 1.64 & 2.79 & 2.44 \\
\hline
\end{tabular}

CR - Crop residue $\quad$ SB - Phosphate Solubalizing Bacteria $\quad$ CFU - Colony Farming units FPs - Fluorescent Pseudomonas

Table.4 Soil microbial population at grand growth period of rabi sunflower as influenced by tank silt + crop residue application and row spacing under set row cultivation (Pooled over 3 years)

\begin{tabular}{|c|c|c|c|c|c|c|c|c|c|c|c|c|}
\hline \multirow[b]{2}{*}{ Treatment } & \multicolumn{4}{|c|}{ Bacteria (CFU/g of soil X 107 $)$} & \multicolumn{4}{|c|}{ Fungi (CFU/g of soil X $\left.10^{4}\right)$} & \multicolumn{4}{|c|}{ Actionmycetes (CFU/g soil X 10 ${ }^{4}$ ) } \\
\hline & $\begin{array}{c}\text { 2004- } \\
05\end{array}$ & $\begin{array}{c}\text { 2005- } \\
06\end{array}$ & $\begin{array}{c}\text { 2006- } \\
07\end{array}$ & Pooled & $\begin{array}{c}\text { 2004- } \\
05\end{array}$ & $\begin{array}{c}\text { 2005- } \\
06\end{array}$ & $\begin{array}{c}\text { 2006- } \\
07\end{array}$ & Pooled & $\begin{array}{c}\text { 2004- } \\
05\end{array}$ & $\begin{array}{c}2005- \\
06\end{array}$ & $\begin{array}{c}\text { 2006- } \\
07\end{array}$ & Pooled \\
\hline Farmers' practice $(35 \mathrm{~cm})$ & 12.56 & 9.44 & 14.38 & 12.13 & 8.33 & 7.18 & 9.30 & 8.27 & 6.43 & 5.58 & 7.93 & 6.65 \\
\hline Recommended Practice $(60 \mathrm{~cm})$ & 14.22 & 11.66 & 15.31 & 13.73 & 10.06 & 9.39 & 11.33 & 10.26 & 8.36 & 6.77 & 9.76 & 8.30 \\
\hline Tank silt + CR in set furrows $(135 \mathrm{~cm})$ & 23.85 & 19.33 & 27.90 & 23.69 & 16.91 & 15.88 & 18.89 & $\mathbf{1 7 . 2 3}$ & 12.92 & 10.70 & 14.49 & $\mathbf{1 2 . 7 0}$ \\
\hline Tank silt + CR in set furrows $(45-90-45 \mathrm{~cm})$ & 25.47 & 19.67 & 29.16 & 24.77 & 18.28 & 16.44 & 20.32 & 18.35 & 14.06 & 11.15 & 16.01 & 13.74 \\
\hline Tank silt + CR in set furrows $(45-135-45 \mathrm{~cm})$ & 24.76 & 20.03 & 28.43 & 24.41 & 17.66 & 17.85 & 19.06 & 18.19 & 13.33 & 11.01 & 15.50 & 13.28 \\
\hline Flatbed $(135 \mathrm{~cm})$ & 16.93 & 13.98 & 18.87 & 16.59 & 11.67 & 10.24 & 13.90 & 11.94 & 9.46 & 7.83 & 10.67 & 9.32 \\
\hline Flatbed $(45-90-45 \mathrm{~cm})$ & 18.30 & 15.22 & 20.10 & 17.87 & 12.37 & 11.33 & 15.03 & 12.91 & 10.16 & 8.70 & 11.28 & $\mathbf{1 0 . 0 5}$ \\
\hline Flatbed $(45-135-45 \mathrm{~cm})$ & 17.36 & 14.78 & 19.73 & 17.29 & 12.29 & 10.43 & 14.17 & 12.30 & 9.68 & 8.56 & 10.82 & 9.68 \\
\hline $\mathrm{S} . \mathrm{Em} \pm$ & 0.86 & 0.94 & 0.93 & 0.64 & 0.80 & 0.71 & 0.71 & 0.43 & 0.51 & 0.59 & 0.58 & 0.30 \\
\hline C.D at $\overline{5 \%}$ & 2.61 & 2.84 & 2.83 & 1.84 & 2.43 & 2.15 & 2.14 & 1.22 & 1.54 & 1.79 & 1.75 & 0.92 \\
\hline
\end{tabular}

$\mathrm{CR}-$ Crop residue

CFU - Colony Farming Units 
Table.5 Soil microbial population at grand growth period of rabi sunflower as influenced by tank silt + crop residue application and Row spacing under set row cultivation (Pooled over 3 years)

\begin{tabular}{|c|c|c|c|c|c|c|c|c|c|c|c|c|}
\hline \multirow[b]{2}{*}{ Treatment } & \multicolumn{4}{|c|}{ "Azotobacter (CFU/g soil X 10 $\left.{ }^{4}\right)$} & \multicolumn{4}{|c|}{ PSB (CFU/g soil X 10 $\left.{ }^{4}\right)$} & \multicolumn{4}{|c|}{ FPs (CFU/g soil X 10 $\left.{ }^{4}\right)$} \\
\hline & $\begin{array}{c}\text { 2004- } \\
05\end{array}$ & $\begin{array}{c}2005- \\
06\end{array}$ & $\begin{array}{l}\text { 2006- } \\
07\end{array}$ & Pooled & $\begin{array}{l}\text { 2004- } \\
05\end{array}$ & $\begin{array}{c}2005- \\
06\end{array}$ & $\begin{array}{l}\text { 2006- } \\
07\end{array}$ & Pooled & $\begin{array}{l}\text { 2004- } \\
05\end{array}$ & $\begin{array}{c}2005- \\
06\end{array}$ & $\begin{array}{l}\text { 2006- } \\
07\end{array}$ & Pooled \\
\hline Farmers' practice $(35 \mathrm{~cm})$ & 8.50 & 7.48 & 8.82 & 8.27 & 12.33 & 10.44 & 13.78 & 12.18 & 10.33 & 9.66 & 12.51 & 10.84 \\
\hline Recommended practice $(60 \mathrm{~cm})$ & 9.83 & 9.65 & 10.75 & 10.08 & 15.66 & 13.73 & 19.03 & 16.14 & 13.30 & 12.32 & 15.06 & 13.56 \\
\hline Tank silt + CR in set furrows $(135 \mathrm{~cm})$ & 17.89 & 16.63 & 19.69 & 18.07 & 28.33 & 23.86 & 30.05 & 27.41 & 23.89 & 20.79 & 26.48 & 23.72 \\
\hline $\begin{array}{l}\text { Tank silt + CR in set furrows }(45-90-45 \\
\mathrm{cm})\end{array}$ & 19.67 & 18.00 & 21.63 & 19.77 & 30.60 & 25.67 & 32.67 & 29.65 & 25.00 & 22.04 & 28.39 & 25.14 \\
\hline $\begin{array}{l}\text { Tank silt }+ \text { CR in set furrows }(45-135-4 \\
\mathrm{cm})\end{array}$ & 18.33 & 17.66 & 20.61 & 18.87 & 29.11 & 24.70 & 31.84 & 28.55 & 24.21 & 21.61 & 27.34 & 24.39 \\
\hline Flatbed $(135 \mathrm{~cm})$ & 11.34 & 11.86 & 15.70 & 12.97 & 20.76 & 16.74 & 24.68 & 20.73 & 15.70 & 13.68 & 19.22 & 16.20 \\
\hline Flatbed $(45-90-45 \mathrm{~cm})$ & 13.67 & 12.67 & 17.03 & 14.46 & 22.17 & 18.00 & 26.80 & 22.32 & 17.33 & 15.37 & 21.08 & 17.93 \\
\hline Flatbed $(45-135-45 \mathrm{~cm})$ & 13.08 & 13.11 & 16.71 & 14.30 & 21.10 & 17.88 & 25.97 & 21.65 & 16.44 & 14.63 & 19.67 & 16.92 \\
\hline S. Em \pm & 0.65 & 1.04 & 1.21 & 0.57 & 0.99 & 0.93 & 1.37 & 0.92 & 0.70 & 0.91 & 1.21 & 0.80 \\
\hline C.D at $5 \%$ & 1.98 & 3.15 & 3.67 & 1.64 & 3.00 & 2.81 & 4.15 & 2.79 & 2.13 & 2.76 & 3.68 & 2.44 \\
\hline
\end{tabular}


The pooled analysis indicated that, application of tank silt + crop residue in set furrows with wider row spacing $(135 \mathrm{~cm})$ recorded the highest sunflower yield (717 $\mathrm{kg} / \mathrm{ha}$ ) followed by tank silt + crop residue in set furrows with paired row planting of 45-90$45 \mathrm{~cm}$ and 45-135-45 $\mathrm{cm}(674$ and $694 \mathrm{~kg} / \mathrm{ha}$, respectively) and were significantly higher over farmers practice $(312 \mathrm{~kg} / \mathrm{ha})$ (Table 1$)$. Binita (2006) and Annadurai et al., (2005) revealed that tank silt as amendment enhanced the production of different crops. The increase in sunflower yield with tank silt + crop residue in set furrow having wider spacing $(135 \mathrm{~cm})$, paired row spacing of 45 90-45 $\mathrm{cm}$ and $45-135-45 \mathrm{~cm}$ over farmers practice was 130,116 and 122 per cent, respectively. The increased yield may be due to higher soil moisture and nutrient availability in the soil. Tank silt increased the soil moisture content and nutrient availability in the soil. The results are in conformity with the findings of Anonymous (2005). According to them application of tank silt in set furrows increased the sunflower yield by 55 per cent over farmers practice. Again they reiterated that maximum grain yield of sunflower was obtained under set row cultivation with wider row spacing $(135 \mathrm{~cm})$ as compared to farmers practice.

\section{Microbial population}

In the present study, considerable improvement in the population of soil microorganisms was noticed. Application of tank silt + crop residue in set furrows with paired row spacing of 45-90-45 cm registered significantly higher population of bacteria (24.77 CFU/g of soil $\left.\mathrm{X} 10^{7}\right)$, fungi (18.35 $\mathrm{CFU} / \mathrm{g}$ of soil $\mathrm{X} 10^{4}$ ), actinomycetes (13.74 $\mathrm{CFU} / \mathrm{g}$ of soil $\left.\mathrm{X} 10^{4}\right)$, azotobacter $(19.77$ CFU/g of soil X10 ${ }^{4}$ ), PSB (29.65 CFU/g of soil $\left.\mathrm{X} 10^{4}\right)$ and FPs $(25.14 \mathrm{CFU} / \mathrm{g}$ of soil $\left.\mathrm{X} 10^{4}\right)$ as compared to the other treatments. However, it was on par with tank silt + crop residue application in set furrows with wider and paired row spacing of $135 \mathrm{~cm}$ and $45-$ $135-45 \mathrm{~cm}$. The increase in population was 104, 122, 107, 139, 147 and 132 per cent, respectively over farmers' practice (Table 4 and 5). Improvement in the soil organic carbon content by addition of tank silt and respective crop residues in set furrows with wider planting pattern might have played vital role in enhancing microbial activity. Angers et al., (1995) were of the opinion that, dynamics of soil organic matter and microbial biomass were most important and had greater influence on soil fertility enrichment. Incorporation of crop residues might stimulated the populations of nitrogen fixing bacteria viz., Azotobacter and Rhizopus sp., bacteria and actionmycetes bacteria, actinomycetes, fungi and ammonifying bacterial population in the soil (Mukharjee et al., 1990). Hence it may conclude that, Application of tank silt + crop residue in set furrows with either wider or paired row planting was found to be optimum to improve pearlmillet $(1700$ and $1737 \mathrm{~kg} / \mathrm{ha}$, respectively) and sunflower yield (717 and $694 \mathrm{~kg} / \mathrm{ha}$, respectively) and it also helps in improve the crop and soil productivity of Vertic-Inceptisol.

\section{References}

Angres, D. A., Voroney, R. P. and Dose, D., 1995, Dynamics of soil organic matter and corn residues affected by tillage practices. Soil Sci. Soc. American J., 59 (5): 1311-1315.

Annadurai, B., Arunachalam, N. and Mahalingam, 2005, Effect of tank silt and press mud mixture amended on the physical properties. J. Soils and Crops, 15 (1): 26-29.

Anonymous, 1998, Annu. Rep. (1997-98), All India Co-ordinated Research Project for Dryland Agriculture, Bijapur, pp. 44-54. Anonymous, 2005, Annu. Rep. (2004-05), All 
India Co-ordinated Research Project for Dryland Agriculture, Bijapur, pp. 175181.

Binita, N. K., 2006, Characterization of tank silt on northern Karnataka and evaluation of the effect of tank silt growth and yield of groundnut. Ph. D. Thesis, Uni. Agric. Sci., Dharwad.

Guled, M. B., Lingappa, S., Itnal, C. J., Shirahatti, M. S. and Yaranal, R. S., 2003, Resource conversation and management in rainfed agro-ecosystem - A compendium far research and Development. Techn. Bull., 34 : 15-18.

Keshavamurthy, S. V. and Kotur, S. C., 1996, A comparison of tank silt, FYM and SSP as a source of $\mathrm{P}$ to Ney Poovan banana. Extend. Sum. Intern. Conf. Managing Natural Reso., p. 238.

Manna, M. C. and Ganguly, T. K., 2001, Influence of FYM and fertilizer $\mathrm{N}$ on soil microbial biomass dynamics, under soybean-wheat-fallow system. Indian $J$. Agric. Res., 35: 48-51.

Mishra, P. K., 2002, Indigenous Technical
Knowledge on Soil and Water Conservation in Semi-Arid India, published by Agro-ecosystem Directorate (rainfed), NATP, CRIDA, Hyderabad, pp. 1-150.

Mukharjee, D., Ghosh, S. L. and Das, A. C., 1990, A study on the chemical and microbiological changes in soil. Indian Agrist., 34: 1-10.

Ramesh, N. R., 2001, Characterization of tank sediments of Dharwad district. M. Sc. (Agri.) Thesis, Uni. Agric. Sci., Dharwad.

Seastedt, T. R., 1984, The role of microorthropods in decomposition and mineralization process. Annu. rev. Ent., 29: 25-46.

Tian, G., Brulsarad, L. and Kang, B. T., 1995, Breakdown of plant residues with contrasting chemical compositions under humid tropical conditions, effects of earthworms and millipedes. Soil Bio. Bio-chem., (United Kingdom), 27: 277280.

\section{How to cite this article:}

Yadahalli G. S., M. B. Guled and Surakod V. S. 2017. Influence of Set Row Cultivation on Pearlmillet-Sunflower Sequence Cropping System in Vertic-Inceptisol. Int.J.Curr.Microbiol.App.Sci. 6(7): 4576-4584. doi: http://dx.doi.org/10.20546/ijcmas.2017.607.478 